\title{
Magnetospirillum gryphiswaldense
}

Dirk Schüler ${ }^{1}$, Caroline L. Monteil ${ }^{2,3} \&$ Christopher T. Lefevre ${ }^{2, *}$

${ }^{1}$ Dept. of Microbiology, University of Bayreuth, Bayreuth, Germany

${ }^{2}$ CNRS, CEA, Aix-Marseille Université, UMR7265 Biosciences and Biotechnologies Institute of Aix-Marseille, Saint Paul lez Durance, France

${ }^{3}$ Sorbonne Université, UMR CNRS7590, Muséum National d'Histoire Naturelle, IRD, Institut de Minéralogie, de Physique des Matériaux et de Cosmochimie, Paris, France

*Correspondence: christopher.lefevre@cea.fr

Keywords: magnetotaxis, magnetosome, biomineralization, magnetite 


\section{Summary}

Magnetospirillum gryphiswaldense is amongst the best studied representatives of magnetotactic bacteria. These microbes use the Earth's magnetic field for navigation thanks to unique organelles, the magnetosomes, which are membrane-enveloped, iron-rich magnetic particles. Because it can be grown in the lab relatively easily and is genetically tractable, $M$. gryphiswaldense has been subject of many studies on magnetotaxis, organelle biosynthesis and biomineralization in prokaryotes and used for environmental and medical applications. Magnetosome biosynthesis starts with the formation of empty membrane vesicles, in which large amounts of iron is transported and mineralized as magnetite $\left(\mathrm{Fe}_{3} \mathrm{O}_{4}\right)$. Chains of nascent magnetosome particles are concatenated, positioned and segregated by a multi-partite cytoskeletal network ("magnetoskeleton"). The >30 most relevant proteins controlling magnetosome biosynthesis are encoded by large gene clusters within a $100 \mathrm{~kb}$ genomic "magnetosome island".

\section{Key Facts}

Isolated from sediment of the river Ryck near the town of Greifswald (Latin: "Gryphiswaldia") in Germany in 1990 by D. Schüler, it was one of the first magnetotactic bacteria recovered in culture.

The founding species of the genus Magnetospirillum that comprises several other well-studied magnetotactic species but also non-magnetotactic members.

Spiral-shaped Gram-negative bacterium, producing 20-50 intracellular magnetite crystals arranged in a chain.

Microaerophilic, facultative anaerobe using oxygen or nitrate as respiratory electron acceptors. Tolerates atmospheric oxygen for growth, but forms magnetosomes only in suboxic conditions.

Highly motile by two polar flagella. Magneto-aerotactic swimming polarity is controlled by oxygen sensing through one of the most complex chemosensory networks known in bacteria.

Entire magnetosome biosynthesis pathway has been genetically transplanted from $M$. gryphiswaldense to non-magnetotactic bacteria. 
Amenable to mass cultivation and production of magnetic nanoparticles. Living bacteria and engineered and functionalized magnetosomes are being studied for use in applications including magnetic nanorobots, biocatalysts, probes for drug delivery, magnetic resonance imaging and cancer therapies.

\section{Taxonomic and Classification information}

KINGDOM: Bacteria

PHYLUM: Proteobacteria

CLASS: Alphaproteobacteria

ORDER: Rhodospirillales

FAMILY: Rhodospirillaceae

GENUS: Magnetospirillum

SPECIES: gryphiswaldense

\section{References}

1. Gandia, D. et al. (2019) Unlocking the potential of magnetotactic bacteria as magnetic hyperthermia agents. Small 15, e1902626

2. Kolinko, I. et al. (2014) Biosynthesis of magnetic nanostructures in a foreign organism by transfer of bacterial magnetosome gene clusters. Nat. Nanotechnol. 9, 193-197

3. Mickoleit, F, Lanzloth, C. and Schüler D. (2020) A versatile toolkit for controllable and highly selective multifunctionalization of bacterial magnetic nanoparticles. Small doi: $10.1002 / \mathrm{smll} .201906922$

4. Monteil, C.L. et al. (2020) Repeated horizontal gene transfers triggered parallel evolution of magnetotaxis in two evolutionary divergent lineages of magnetotactic bacteria. ISME J. doi: 10.1038/s41396-020-0647-x 
5. Popp, F. et al. (2014) Polarity of bacterial magnetotaxis is controlled by aerotaxis through a common sensory pathway. Nat. Commun. 5, 5398

6. Scheffel, A. et al. (2006) An acidic protein aligns magnetosomes along a filamentous structure in magnetotactic bacteria. Nature 440, 110-114

7. Schleifer, K.H. et al. (1991) The genus Magnetospirillum gen. nov. description of Magnetospirillum gryphiswaldense sp. nov. and transfer of Aquaspirillum magnetotacticum to Magnetospirillum magnetotacticum comb. nov. Syst. Appl. Microbiol. 14, 379-385

8. Schübbe, S. et al. (2003) Characterization of a spontaneous nonmagnetic mutant of Magnetospirillum gryphiswaldense reveals a large deletion comprising a putative magnetosome island. J. Bacteriol. 185, 5779-5790

9. Toro-Nahuelpan, M. et al. (2019) MamY is a membrane-bound protein that aligns magnetosomes and the motility axis of helical magnetotactic bacteria. Nat. Microbiol. 4, 19781989

10. Raschdorf, O. et al. (2016) Genetic and ultrastructural analysis reveals the key players and initial steps of bacterial magnetosome membrane biogenesis. PLoS Genet. 12, e1006101

\section{Acknowledgement}

Work in the Schüler laboratory is supported by the Deutsche Forschungsgemeinschaft (Grant No. Schu 1080/9-2), the German BMBF (Grant No. 031B0849) and the European Research Council (ERC) under the European Union's Horizon 2020 research and innovation program (Grant No. 692637 to D.S.). Work in the Lefevre laboratory is supported by the French National Agency for Research (SIGMAG: ANR-18-CE31-0003 and PHOSTORE: ANR-19-CE010005-02). Some of the images were provided/recorded by Claus Lang (fluorescence micrograph), Mauricio Toro-Nahuelpan (tomograph) and Karen Tavares (colonies). 